\title{
Metastatic Renal Cell Carcinoma in the Thyroid Gland
}

\author{
Mark Cruz ${ }^{1}$, Gregory Schmidt ${ }^{1}$, Jeptha Johnson ${ }^{1}$, Mohamed Shakir ${ }^{1}$, and Thanh Hoang ${ }^{1}$ \\ ${ }^{1}$ Walter Reed National Military Medical Center
}

April 28, 2020

\begin{abstract}
Thyroid nodules are often incidentally noted on imaging studies with an estimated incidence of $25 \%$ on neck CT scan. The presence of a PET-avid thyroid nodule, especially in a patient with a history of malignancy, should prompt evaluation with fine-needle aspiration to evaluate for malignancy given the high probability.
\end{abstract}

Title Page

Clinical Picture:

Metastatic Renal Cell Carcinoma in the Thyroid Gland

Mark M. Cruz, MD ${ }^{1}$

Gregory S. Schmidt, $\mathrm{MD}^{1}$

Jeptha T. Johnson, MD ${ }^{2}$

Thanh D. Hoang, $\mathrm{DO}^{1}$

Mohamed KM. Shakir, MD ${ }^{1}$

(1) Division of Endocrinology, Department of Medicine

(2) Department of Pathology

Walter Reed National Military Medical Center

8901 Rockville Pike, Bethesda, Maryland 20889

Corresponding Author: Dr. Thanh D. Hoang

Division of Endocrinology, Walter Reed National Military Medical Center, 8901 Wisconsin Ave,

Bethesda, MD 20889.

Email: thanh.d.hoang.mil@mail.mil

Disclaimer: The views expressed in this article are those of the authors and do not reflect the official policy of the Department of Army/Navy/Air Force, Department of Defense, or the U.S. Government.

Key Clinical Message:

Thyroid nodules are often incidentally noted on imaging studies with an estimated incidence of $25 \%$ on neck CT scan. The presence of a PET-avid thyroid nodule, especially in a patient with a history of malignancy, 
should prompt evaluation with fine-needle aspiration to evaluate for possible malignancy given the high pretest probability.

\section{Key words:}

RCC, metastatic disease, PET-CT, fine-needle aspiration, thyroid

\section{Clinical vignette:}

83-year-old man presented for evaluation of non-toxic multinodular goiter. Medical history was significant for renal cell carcinoma (RCC) metastatic to the hip treated with chemotherapy. A surveillance PETCT demonstrated an FDG-avid right-sided thyroid nodule with SUV of 4.58 (Fig 1). Thyroid ultrasound revealed a $2.2 \mathrm{~cm}$ hypoechoic nodule in the right mid-lobe with faint microcalcifications (Fig 2 ). The patient denied dysphagia, neck radiation, or family history of thyroid cancer. Thyroid function tests were normal. FNA showed cohesive groups of cells with large nuclei, irregular nuclear contours, granular chromatin with "champagne bubble cytoplasm" (Fig 3). Subsequent thyroidectomy specimen was positive staining for RCC marker and PAX8 by immunohistochemistry, confirming metastatic RCC.

Thyroid nodules are often incidentally found on imaging studies with an estimated $25 \%$ incidence on neck CT scan (1). The risk of malignancy in thyroid nodules identified on PET-CT is reported 27.8-74.0\%, and nodules with higher SUVs have a higher likelihood of malignancy (1). The overall incidence of metastatic disease to the thyroid gland is approximately $2 \%$ in autopsy series (2). The most common primary malignancy in cases of thyroid metastases is RCC followed in descending order by lung, gastrointestinal, and breast malignancies. In our patient, both PET-avidity and presence of microcalcifications in the thyroid nodule suggested a high risk of malignancy.

This case highlights the importance of high clinical suspicion in patients with PET-avid thyroid nodules, especially those with a history of malignancy. Such patients should undergo FNA to evaluate for malignancy given the high pre-test probability.

\section{Authors contribution statement:}

Mark M. Cruz, MD - Author

Gregory S. Schmidt, MD- Reviewer

Jeptha Johnson, MD - Pathologist, provided the slides, reviewer

Thanh D. Hoang, DO- Reviewer

Mohamed KM Shakir - Reviewer

\section{References:}

(1) Chun, A. R., Jo, H. M., Lee, S. H., Chun, H. W., Park, J. M., Kim, K. J., .. Kim, B. Y. (2015). Risk of Malignancy in Thyroid Incidentalomas Identified by Fluorodeoxyglucose-Positron Emission Tomography. Endocrinology and Metabolism, 30(1), 71.

(2) Nixon, I. J., Coca-Pelaz, A., Kaleva, A. I., Triantafyllou, A., Angelos, P., Owen, R. P., Ferlito, A. (2016). Metastasis to the Thyroid Gland: A Critical Review. Annals of Surgical Oncology, 24(6), 1533-1539.

\section{Abbreviations:}

RCC: renal cell carcinoma

PET-CT: positron emission tomography - computed tomography

FDG: fluorodeoxyglucose

SUV: standard uptake value

FNA: fine needle aspiration 


\section{Figures Legend:}

Figure 1: PET-CT demonstrating an FDG-avid right-sided thyroid nodule with a standard uptake value of 4.58

Figure 2: Thyroid ultrasound with a $2.2 \mathrm{~cm}$ hypoechoic nodule in the right middle lobe with faint microcalcifications

Figure 3: A cytology smear of the FNA demonstrating cohesive groups of cells with large nuclei, irregular nuclear contours, granular chromatin with finely vacuolated cytoplasm "champagne bubble cytoplasm"

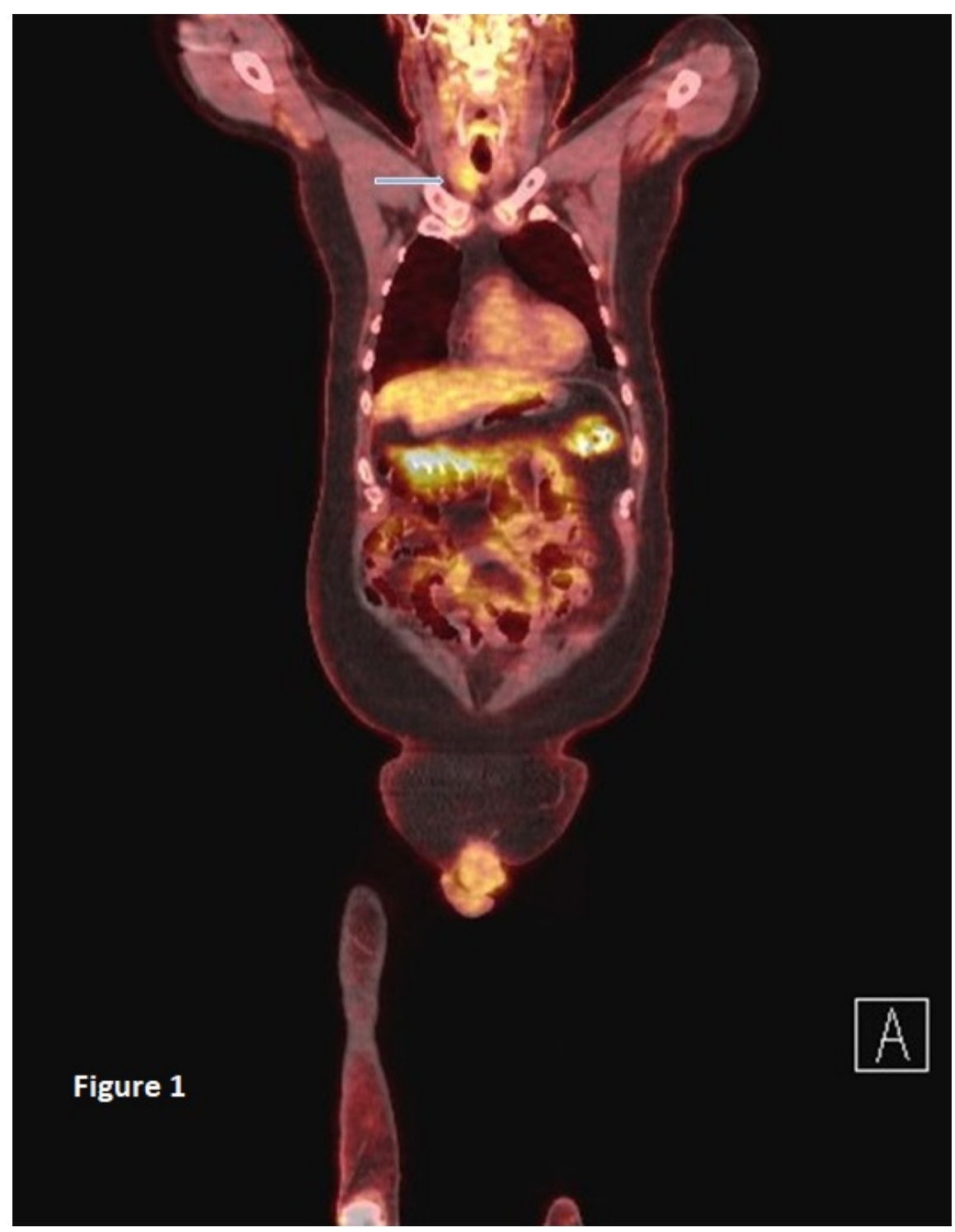



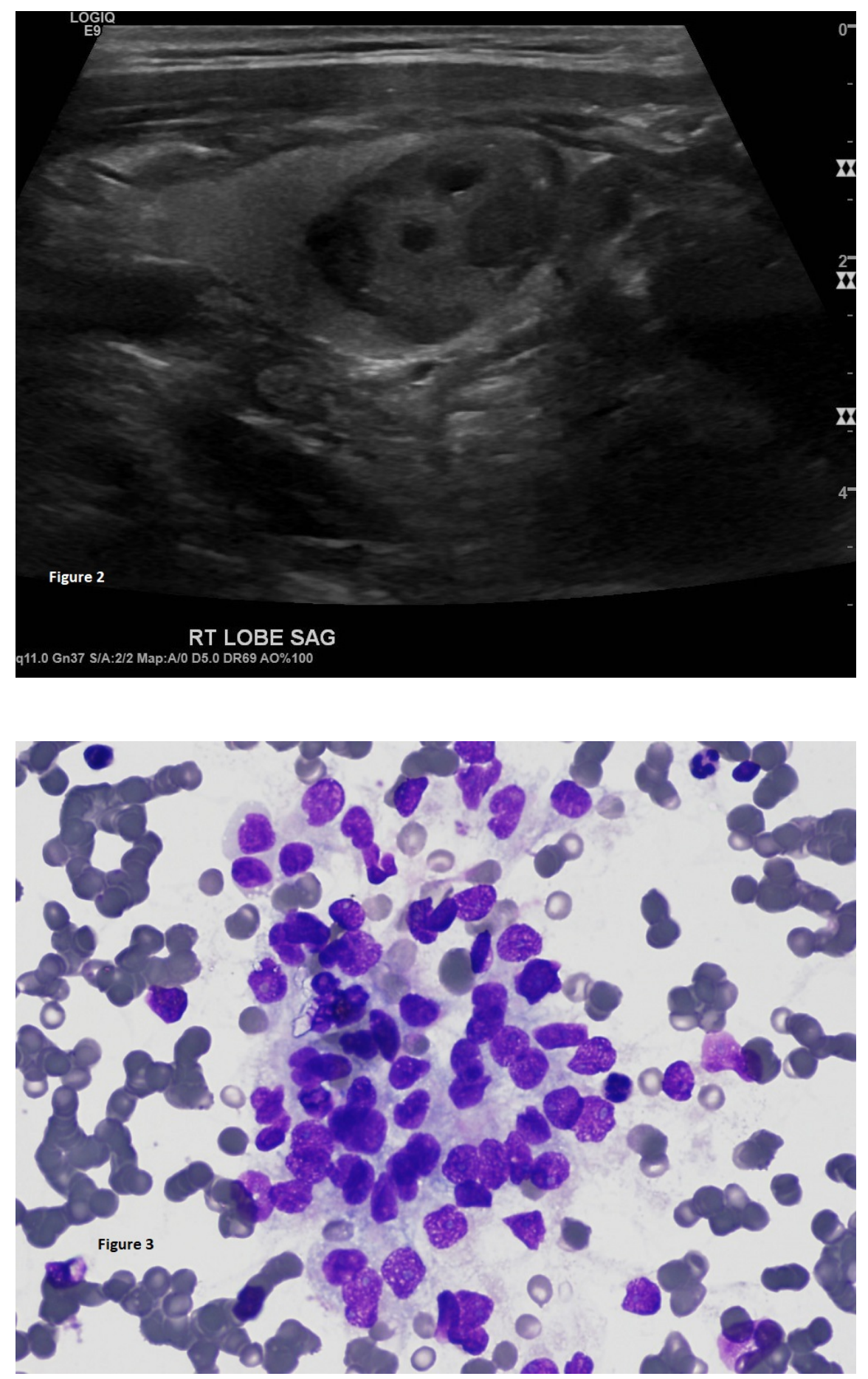